\title{
APPLYING SWARM INTELLIGENCE TO DESIGN THE RECONFIGURABLE FLOW LINES
}

\author{
Goyal, K. K. ; Jain, P. K.* \& Jain, M.** \\ * Mechanical and Industrial Engineering Department, IIT Roorkee, Roorkee-247667, India \\ ${ }^{* * *}$ Department of Mathematics, IIT Roorkee, Roorkee-247667, India \\ E-Mail:kkg74dme@iitr.ernet.in,kapilacad@gmail.com, pjainfme@ iitr.ernet.in, \\ madhufma@iitr.ernet.in
}

\begin{abstract}
Reconfigurable Manufacturing System (RMS) justifies the need of hour by combining the high throughput of dedicated manufacturing system with the flexibility of flexible manufacturing systems. At the heart of RMS lies the Reconfigurable Machine Tools which are capable of performing multiple operations in its existing configurations and can further be reconfigured into more configurations which makes the configuration selection an arduous task. In the present research work the design of single part reconfigurable flow line has been attempted considering multiple objectives i.e. cost and machine utilization. A methodology is proposed for multiple objective optimization of RMS configuration based on machine utilization and cost by applying Multiple Objective Particle Swarm Optimization (MOPSO). A case study has been taken to illustrate the developed approach of flow line optimization applying MOPSO.

(Received in February 2012, accepted in August 2012. This paper was with the authors 1 month for 1 revision.)
\end{abstract}

Key Words: Reconfigurable Manufacturing System, Reconfigurable Machine Tool, Single Part Reconfigurable Flow Line, Machine Selection, Multiple Objective Particle Swarm Optimization

\section{INTRODUCTION}

The increasingly fluctuating and uncertain demands coupled with the ever changing products and process technologies have pronounced the inability of conventional manufacturing systems to handle the modern challenges efficiently and cost effectively.

Considering the limitations of existing manufacturing systems in terms of adjusting the capacity and functionality economically and rapidly, has paved the way for a new manufacturing paradigm. Moreover the existing systems are facing a threat of obsolescence due to the fast pace of development in the enabling technologies and the ever changing needs of the customers. Therefore, there is an acute need of a manufacturing system, which is responsive to the market requirements and can easily be upgraded. The responsiveness of the system is the ability of a system to adjust its functionality and capacity rapidly with respect to ever changing product mix and volume. The answer to all these requirements was proposed by Koren [1] in terms of Reconfigurable Manufacturing Systems (RMS).

The concept of 'modular manufacturing' defined by Tsukune et al. is also similar to the Reconfigurable Manufacturing System. Later in 1996 the Engineering Research Centre for Reconfigurable Manufacturing Systems (ERC-RMS) was established at the University of Michigan, Ann Arbor to develop and implement reconfigurable manufacturing systems. Koren et al. (1999) defined RMS as:

"An RMS is designed at the outset for rapid change in its structure, as well as in hardware and software components, in order to quickly adjust the production capacity and functionality 
within a part family in response to sudden unpredictable market changes as well as introduction of new products or new process technology".

The RMS is designed at the outset to offer the exact functionality and capacity, exactly when it is needed. As shown in Fig. 1 [2] RMS is having positive attributes of both the dedicated manufacturing system (DMS) and flexible manufacturing system (FMS).

Reconfiguration allows an RMS to achieve throughput approaching that of a DMS but allows it to produce the whole range of products in the family of parts. Thus the goal of RMS is to provide exactly the capacity and the functionality. Short conversion times between processing of different batches or between two members of a part family within the same day plays a crucial role in the efficiency of RMS. Fig. 2 depicts the configuration of reconfiguration machine tools (RMTs) from the standard module library, in which two machine configurations (i.e. $m c_{1}^{1}$ and $m c_{1}^{2}$ ) of machine one are assembled by just varying the auxiliary modules (AM), while the machine two is shown in its single configuration having different auxiliary modules and basic modules (BM) (i.e. $m c_{2}^{1}$ ).

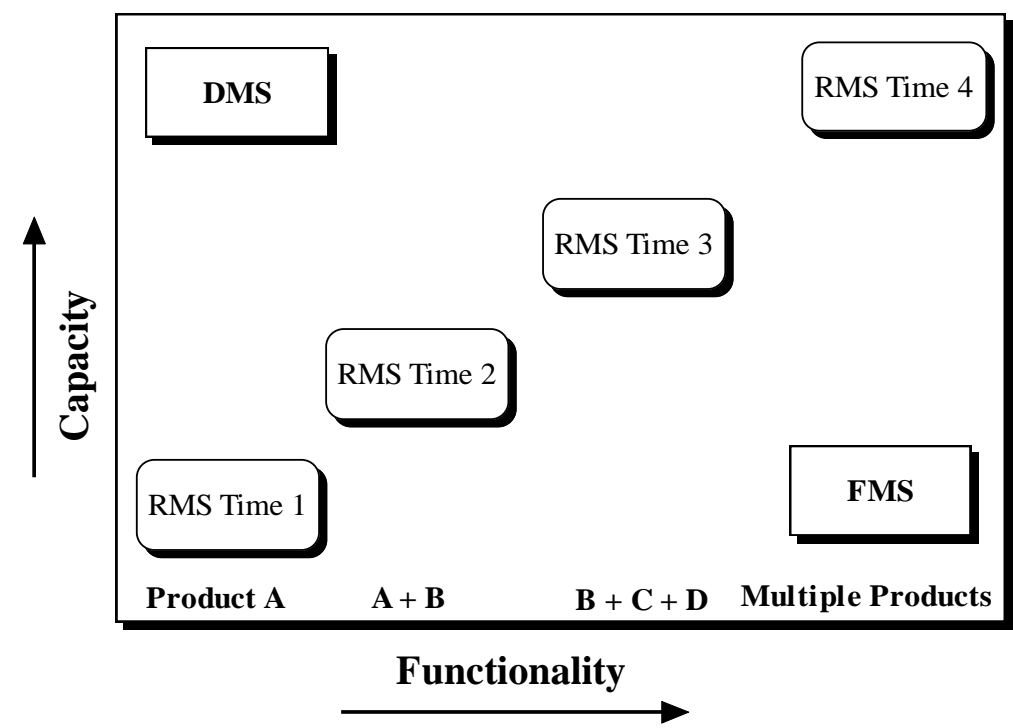

Figure 1: Dynamically adaptable RMS compared with DMS \& FMS (adopted from [1]).

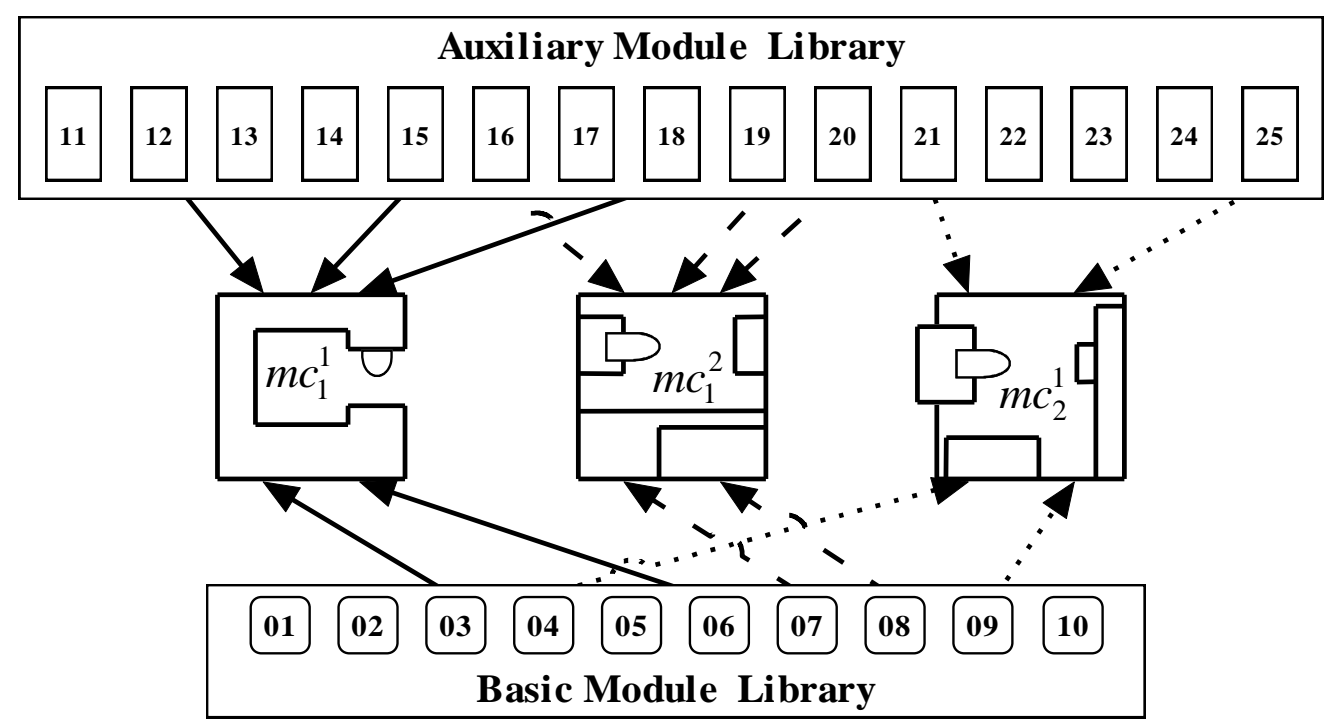

Figure 2: Configuring RMTs through module library. 
In the conventional manufacturing systems (cellular manufacturing system (CMS), dedicated manufacturing system and flexible manufacturing system) the operation sequence required for manufacturing a part is generated after knowing the operational capabilities of the available machine tools but in RMS the process is reverse first of all the operation sequence of parts is generated based on which the RMS configuration is designed through assigning the optimal machine configurations to various operations.

According to Urbani et al. [3] the reconfigurability is the ability of a system to adapt to expected or unexpected demand changes through the changes in the system or system component's structure guaranteeing the efficient use of functionalities. Pattanaik et al. [4] have solved the cellular layout problem with reconfigurable machines. Maier-Speredelozzi et al. [5] presented the system convertibility as the capability of a system to adjust production functionality and presented the system convertibility measures based on the assessment of convertibility of the system configuration, machines and material handling equipment. Gumasta et al. [6] have suggested reconfigurability of the RMS on system level. The flow line layout is most appropriate for RMS and from the literature it is evident that most of the RMS modelling approaches follow the flow line layout [7-12]. Son et al. [10] and Youssef and ElMaraghy [13] have modelled a multiple demand period configuration generation by first recording the $k$ best configurations for all the demand periods based on cost as single performance criterion by applying Genetic Algorithm (GA) and in later stage selected the configurations based on configuration similarity and reconfiguration smoothness. While assigning the RMT configurations to the production stages only objective considered is cost which would lead to missing the RMS configurations having high machine utilization with slightly higher cost. Thus in the present work an attempt has been made to design the single part reconfigurable flow line configuration based on machine utilization and cost through applying the multiple objective particle swarm optimization.

\section{PERFORMANCE MEASURES}

In a reconfigurable manufacturing system, the machines are capable of performing variety of operations in its existing configurations and the reconfigurable machine tools (RMT) can further be reconfigured into other configurations. The different configurations thus can further enhance the functionality and can perform number of operations. In such a scenario the availability of large number of machines to perform a single operation, makes it a combinatorial complex problem to assign the RMTs to various operations in an operation sequence. Machine utilization is an important aspect dictating the efficient and effective execution of a manufacturing system. Thus in the present work an approach has been presented to apply the swarm intelligence into the design of multiple objective single part reconfigurable flow line. The following notations have been considered to implement the developed approach:

$$
\begin{array}{ll}
m c_{i}^{j} & \text { - machine } i(1<i<I) \text { in its } j^{\text {th }}\left(1<j<J_{i}\right) \text { configuration } \\
n_{i}^{j} & \text { - number of machines required to satisfy the demand when machine } i \text { with } j^{\text {th }} \\
& \text { configuration is selected } \\
D & \text { - demand rate } \\
F S_{k} & \text { - a set of feasible alternative machine configurations to perform } k^{\text {th }}(1<k<K) \\
& \text { operation }\left\{\left(i_{1}, j_{1}\right),\left(i_{2}, j_{2}\right), \ldots .\left(i_{f}, j_{f}\right) \ldots \ldots\left(i_{F_{k}}, j_{F_{k}}\right)\right\} .
\end{array}
$$

(Here each feasible alternative $f\left(1<f<F_{k}\right)$ is defined as $\left(i_{f}, j_{f}\right)$, where $i_{f}$ specifies the feasible machine and $j_{f}$ specifies the feasible machine configuration.)

$C M_{i}^{j} \quad$ - cost of machine $i$ with $j^{\text {th }}$ configuration (i.e. $m c_{i}^{j}$ ) 
$P_{i, k}^{j} \quad$ - production rate of machine $i$ with $j^{\text {th }}$ configuration for performing $k^{\text {th }}$ operation

$\delta_{i, k}^{j} \quad-=1$ if operation $k$ can be performed with machine $i$ having its $j^{\text {th }}$ configuration, otherwise 0

$C_{p, q} \quad$ - cost of assigning $p^{\text {th }}$ machine with $q^{\text {th }}$ configuration from the feasible alternative machine configurations to perform an operation at specified demand rate

$M U_{p, q}$ - machine utilization of assigning $p^{\text {th }}$ machine with $q^{\text {th }}$ configuration from the feasible alternative machine configurations to perform an operation at specified demand rate

\subsection{Cost}

Cost is an important performance parameter driving the selection of machine configuration for a particular operation. In the present scenario the manufacturers are facing stiff competition due to globalization and volatile markets. Thus meeting the customer demands economically is most important. The cost $\left(C_{p, q}\right)$ of a feasible alternative machine configurations for performing $k^{\text {th }}$ operation on the specified demand rate is calculated using the Eqs. (1) and (2). The cost is a non-beneficial attribute and is to be minimized.

$$
\begin{gathered}
C_{p, q}=n_{p, q} \times C M_{p}^{q} \\
n_{p, q}=\left\lceil\frac{D}{P_{p, q, k}}\right\rceil
\end{gathered}
$$

where:

$C_{p, q}$ - the cost of $p^{\text {th }}$ machine with $q^{\text {th }}$ configuration to perform $k^{\text {th }}$ operation on the specified demand rate

$n_{p, q} \quad$ - the number of machines required for selected machine configuration

$D \quad$ - the demand rate of the product

$C M_{p}^{q}$ - the cost of machine $p$ with $q^{\text {th }}$ configuration

$P_{p, q, k} \quad$ - the production rate of $p^{\text {th }}$ machine in its $q^{\text {th }}$ configuration for performing operation $k$

The ratio $\frac{D}{P_{p, q, k}}$ is rounded off to the higher integer as number of machines can't be a fraction.

\subsection{Machine utilization (MU)}

The need of higher product variety requests for more production resources, which dictates the higher utilization of resources. In such circumstances utilization of the manufacturing system capacity is very crucial for the mere sustenance and growth of the enterprise. Therefore the system should be utilized to the maximum possible extent by optimizing the system configuration. In the reconfigurable manufacturing environment the availability of multifunctional machines which can further be reconfigured into various configurations turns the machine selection problem into combinatorial problem. Thus, while selecting the system configuration for a part the machine utilization should be given due consideration. Machine utilization of a machine configuration can be determined as:

$$
M U_{p, q}=\frac{P_{p, k}^{q} \times n_{p}^{q}}{D}
$$




\section{DESIGNING RECONFIGURABLE FLOW LINE APPLYING MOPSO}

In the present work multiple objective particle swarm optimization is applied to get the Pareto frontiers for the problem of designing the single part reconfigurable flow line based on cost and machine utilization. Kennedy and Eberhart [14] first introduced the PSO method driven by the social behaviour of organisms such as fish (schooling) and bird (flocking). During flight, each particle adjusts its position according to its own experience, and the experience of neighbouring particles.

Assume that the search space is $D$-dimensional, and the $i^{\text {th }}$ particle of the swarm can be represented by a $D$-dimensional vector $X_{\mathrm{i}}=\left(x_{\mathrm{i} 1}, x_{\mathrm{i} 2}, \ldots, x_{\mathrm{iD}}\right)$. The velocity of this particle is represented by another $D$-dimensional vector $V_{\mathrm{i}}=\left(v_{\mathrm{i} 1}, v_{\mathrm{i} 2}, \ldots, v_{\mathrm{iD}}\right)$. The best experience of $i^{\text {th }}$ particle (Pbest) is denoted as $P_{\mathrm{i}}=\left(p_{\mathrm{i} 1}, p_{\mathrm{i} 2}, \ldots, p_{\mathrm{iD}}\right)$. The best particle among all the particles in the group $($ Gbest $)$ is represented by the $P_{\mathrm{g}}=\left(p_{\mathrm{g} 1}, p_{\mathrm{g} 2}, \ldots, p_{\mathrm{gD}}\right)$. The superscripts denote the iteration number and the swarm are globally manipulated according to the following two equations in the single objective basic PSO:

$$
\begin{gathered}
v_{i}^{t+1}(d)=\omega * v_{i}^{t}(d)+c_{1} \times \operatorname{rand}_{1}\left[p_{i}^{t}(d)-x_{i}^{t}(d)\right]+c_{2} \times \operatorname{rand}_{2}\left[p_{g}^{t}(d)-x_{i}^{t}(d)\right] \\
x_{i}^{t+1}(d)=x_{i}^{t}(d)+v_{i}^{t+1}(d)
\end{gathered}
$$

where $v_{i}^{t}(d)$ and $x_{i}^{t}(d)$ represent the current velocity and position of the $d^{\text {th }}$ dimension of the $i^{\text {th }}$ particle respectively and $\operatorname{rand}_{1}$ and $\operatorname{rand}_{2}$ are uniform random numbers in the range [0,1]; $c_{1}, c_{2}$ are positive constants, called acceleration constants, and $\omega$ is the inertia weight. Applications, parameter selection, and modified versions of PSO can be found in Eberhart and Shi [15] and Shi and Eberhart [16]. PSO has been implemented for handling the discrete search space for the scheduling of flow shop [17].

In the basic PSO there is no sharing of information with other particles of the swarm, except that each particle can access the global best. In case of multiple objective optimization the sharing of information among all the particles is very crucial in order to achieve the true Pareto front. There are several strategies for storing non-dominated solutions and selecting one to calculate the next velocities and positions. Some authors suggest the use of an external repository to store non-dominated solutions $[18,19]$. There are several criteria to select a particle from the repository, and this strategy has the function of maintaining the diversity of solutions. Coello Coello et al. [20] propose a method of choosing the solution from the external repository combining randomness and proximity between solutions, with the goal of promoting diversity and accelerating the convergence. Salazar-Lechuga and Rowe [21] proposed a PSO based multiobjective algorithm that uses an external repository, making the selection of the leader based on the value of fitness sharing. Li [22] has proposed nondominated sorting PSO optimizer, in which various methodologies related to selection of global leaders for the particles are proposed. In their work they propose ranking the Pareto frontiers based on crowding distance and further selecting the global leaders randomly. Various studies have compared the NSGA II with the MOPSO using the NSGA II concept and found the MOPSO better in terms of locating the true Pareto front with good diversity in the reasonable time $[18,22]$.

Therefore in the present work authors have implemented the non-dominated sorting concept of NSGA- II given by Deb et al. [23] with the basic PSO to handle the discrete and discontinuous search space of reconfigurable flow line optimization. The terminologies central to the concept (non-dominated sorting procedure, crowded distance estimation, crowded comparison operator) of NSGA-II, may be referred from Deb [24]. 


\subsection{The proposed MOPSO algorithm}

The algorithm of MOPSO applied in the present problem is as under:

(i) Initialize the particle swarm $P O P$ randomly. (ii) Evaluate each particle based on the multiple objectives. (iii) Assign Pbest $(i$ ) for each particle (memory of the particle) initially as its present solution. (iv) Perform non dominated sorting and save the non-dominated particles in a separate repository $R E P$, also calculate the crowding distance of the particles and sort the non-dominated particles based on the crowding distance and also save the crowding distance in the repository REP. (v) For the fixed number of iterations following steps are repeated.

a) Compute the new Velocity $V(i)$ in all the dimensions according to the Eq. (4) and new position $X(i)$ according to Eq. (5). The Gbest for each particle in the Eq. (4) in the present algorithm is selected by roulette wheel selection applied on the non-dominated solutions in the repository REP based on the crowding distance of the non-dominated solutions. Pbest $(i)$ is the best position of any particle ever achieved. b) Maintain the particles in the search space limits in case they go beyond by generating the feasible solutions. c) Evaluate each particle and perform non-domination sorting. Add the non-dominated solutions to the repository REP. Eliminate the dominated solutions from REP. d) When the current position of $i^{\text {th }}$ particle is better than its Pbest $(i)$, the current position is updated as the Pbest $(i)$. The comparison is done based on the domination between the two. In case none of them dominate, one of them is selected randomly.

The developed performance indices are applied to optimize a single part reconfigurable flow line allowing paralleling of similar machines. As shown in Fig. 3, each operation is assigned to a production stage according to the precedence constraints of the operation sequence and each stage is further assigned a machine type and its configuration number. The optimal assignment of the machine and its configuration to each production stage is realized by MOPSO taking cost and machine utilization as the objectives. For applying MOPSO in the present study, the set of feasible alternative machine configurations $\left(F S_{k}\right)$ for all the operations are recorded by referring Table I. Each element $f$ of set $F S_{k}$ is a combination of two parameters i.e. the machine and the machine configuration. The total number of feasible alternative machine configurations to perform the $k^{\text {th }}$ operation is $F_{k}$. The recording of these configurations for all the operations is necessary for the constraint handling.

\subsection{Objective function, constraint handling and solution mapping}

The number of decision variables involved in the present study is twice (one for the machine type and other for the machine configuration) the number of stages and all the variables in the solution vector are discrete and discontinuous. The basic principle of PSO is to generate the population of random solutions (i.e., particles) flown through a hyper dimensional search space. Each particle in PSO has an adaptable velocity. But in the present case the solutions belong to a combinatorial problem and the feasible solutions are rather sparse which will lead to infeasible population. Therefore to handle the problems mentioned above, a solution coding is proposed along with the constraint handling through decoding of solutions using the feasible alternative machine configurations generated beforehand. The search space dimensions of the present problem is reduced to the number of production stages and on each stage an operation has to be performed according to the operation sequence. A set of feasible alternative machine configurations $F S_{k}$ for each operation is already recorded as shown in Fig. 3. Now each stage is to be assigned with a feasible machine configuration which is mapped in the present study through the solution coding. As shown in Fig. 4, the value of velocity in the first dimension i.e. at the first production stage $(\mathrm{S}-1)$ is 0.15 and operation 3 is to be performed. 


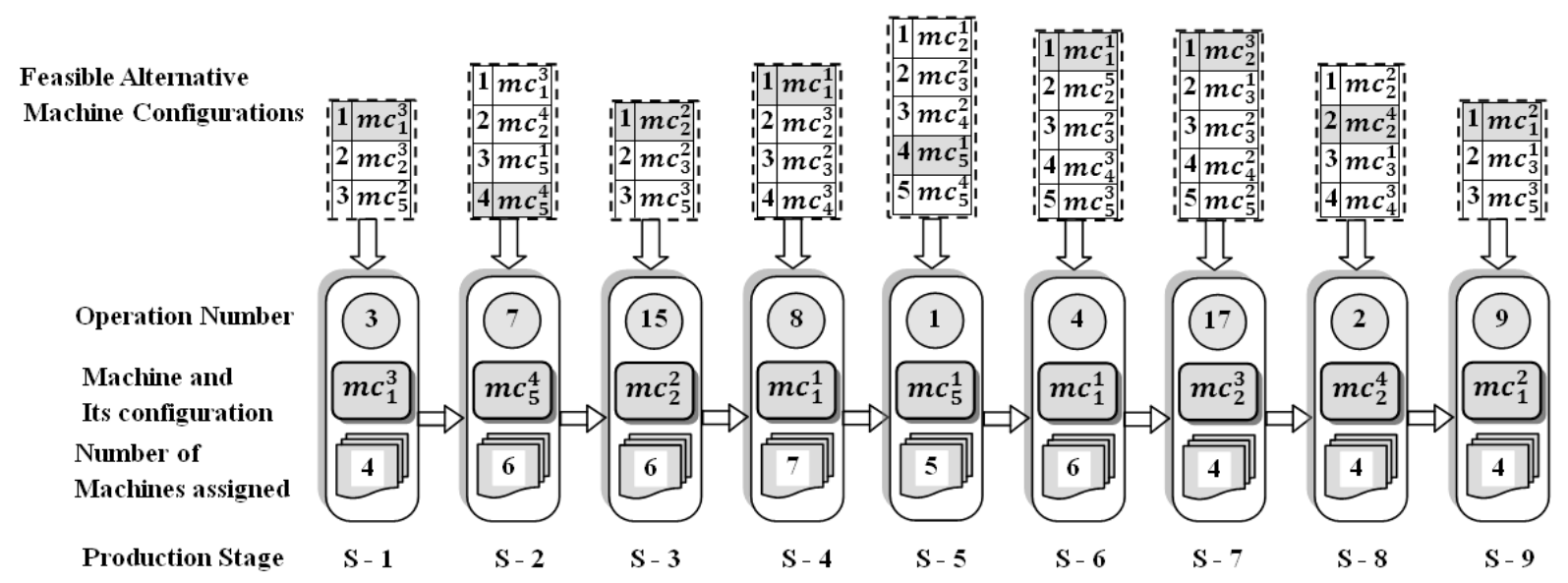

Figure 3: Feasible solution recording for the single part flow line configuration.

Table I. RMT cost and operational capability.

\begin{tabular}{|c|c|c|c|c|c|c|c|c|c|c|c|c|c|c|c|c|c|c|c|c|c|}
\hline \multirow{2}{*}{$\begin{array}{l}\text { Operation } \\
\qquad(k) \rightarrow \\
m c_{i}^{j} \downarrow\end{array}$} & \multicolumn{20}{|c|}{ RMT Production rate in parts/hour for performing various operations } & \multirow{2}{*}{$\begin{array}{c}\text { Cost } \\
\text { (in } 10^{3} \text { of } \\
\text { USD) }\end{array}$} \\
\hline & 1 & 2 & 3 & 4 & 5 & 6 & 7 & 8 & 9 & 10 & 11 & 12 & 13 & 14 & 15 & 16 & 17 & 18 & 19 & 20 & \\
\hline$m c_{1}^{1}$ & - & - & - & 14 & - & - & - & 12 & - & - & - & 8 & - & - & - & 18 & - & - & - & - & 750 \\
\hline$m c_{1}^{2}$ & - & - & - & - & 15 & - & - & - & 20 & - & - & - & - & - & - & - & - & 16 & - & - & 955 \\
\hline$m c_{1}^{3}$ & - & - & 20 & - & - & - & 15 & - & - & - & - & - & - & - & - & 25 & - & - & - & - & 1025 \\
\hline$m c_{1}^{4}$ & - & - & - & - & - & - & - & - & - & 15 & - & - & - & - & - & - & - & - & 12 & - & 840 \\
\hline$m c_{2}^{1}$ & 14 & - & - & - & - & 15 & - & - & - & - & - & 12 & - & - & - & - & - & - & - & 20 & 1215 \\
\hline$m c_{2}^{2}$ & - & 15 & - & - & - & - & - & - & - & - & - & - & 14 & - & 15 & - & - & - & - & - & 910 \\
\hline$m c_{2}^{3}$ & - & - & 25 & - & - & - & - & 18 & - & - & 25 & - & - & - & - & - & 20 & - & - & - & 1140 \\
\hline$m c_{2}^{4}$ & - & 20 & - & - & 20 & - & 18 & - & - & - & - & - & - & 24 & - & - & - & - & - & - & 1350 \\
\hline$m c_{2}^{5}$ & - & - & - & 18 & - & - & - & - & - & - & - & - & 20 & - & - & - & - & 14 & - & 15 & 1050 \\
\hline$m c_{3}^{1}$ & - & 12 & - & - & - & - & - & - & 15 & - & - & 10 & - & - & - & - & 10 & - & - & - & 780 \\
\hline$m c_{3}^{2}$ & 30 & - & - & 26 & - & - & - & 24 & - & - & 24 & - & - & - & 20 & - & 35 & - & 15 & - & 1825 \\
\hline$m c_{4}^{1}$ & - & - & - & - & - & 25 & - & - & - & 30 & - & - & - & - & - & - & - & 25 & - & - & 1350 \\
\hline$m c_{4}^{2}$ & 25 & - & - & - & - & - & - & - & - & - & - & 22 & - & - & - & - & 30 & - & - & 26 & 1500 \\
\hline$m c_{4}^{3}$ & - & 18 & - & 25 & - & - & - & 16 & - & - & - & - & 22 & - & - & 28 & - & - & 20 & - & 1400 \\
\hline$m c_{5}^{1}$ & 16 & - & - & - & - & - & 15 & - & - & - & 15 & - & - & 18 & - & - & - & 18 & - & - & 900 \\
\hline$m c_{5}^{2}$ & - & - & 24 & - & 20 & - & - & - & - & 25 & - & - & - & - & - & - & 24 & - & - & 20 & 1175 \\
\hline$m c_{5}^{3}$ & - & - & - & 24 & - & - & - & - & 30 & - & - & - & - & - & 18 & - & - & - & - & - & 1230 \\
\hline$m c_{5}^{4}$ & 20 & - & - & - & - & 22 & 14 & - & - & - & - & - & - & 20 & - & 16 & - & - & 18 & - & 1175 \\
\hline
\end{tabular}

From Table I the feasible alternative machine configurations $F S_{3}$ can be read as $\left\{m c_{1}^{3}, m c_{2}^{3}, m c_{5}^{2}\right\}$. Thus there are three feasible alternative machine configurations capable of performing operation 3 . Now the particle velocity has to be multiplied by the number of feasible alternative machine configurations $\left(F_{k}\right)$ available to perform the operation, which in this case is three and the final value is to be rounded off to the higher integer. The final value represents the order of the selected machine configuration alternative from the already 
recorded feasible alternatives. In the Fig. 4, the first option is selected from the set of feasible alternative machine configurations which is first machine in its third configuration. The decoding of particle velocities in all the dimensions ensures that for any random value of the particle velocity a feasible machine configuration is always assigned to each production stage. Further alteration of particle velocities is not going to affect the feasibility of the solution due to the mapping schema adopted in the present study. All the feasible alternatives are having equal probability of selection and the search space is also restricted to the feasible region only.

The present study proposes the assignment of machines to all the operations allocated on production stages from the feasible alternative machine configurations based on multiple objectives as given in following equations:

$$
\begin{aligned}
& \text { Minimize } F 1=\sum_{s=1}^{S} C_{p_{s}, q_{s}} \\
& \text { Maximize } F 2=\sum_{s=1}^{S} M U_{p_{s}, q_{s}}
\end{aligned}
$$

Here Eqs. (6-7) represent the fitness function i.e. cost and machine utilization of the configuration selected for the single product flow line. The subscripts $p_{s}$ and $q_{s}$ represent the feasible alternative machine $p$ with its configuration $q$ assigned at the $s^{\text {th }}$ stage.

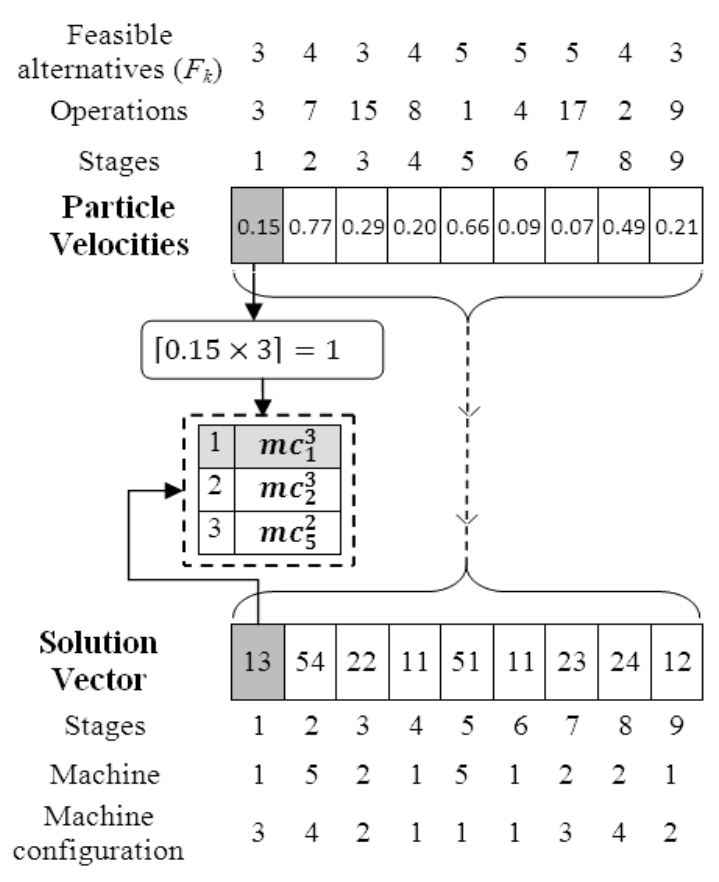

Figure 4: Solution mapping through real coded chromosome.

\section{NUMERICAL ILLUSTRATION}

For illustrating the developed approach of designing single part reconfigurable flow line, a set of RMTs having the operational capabilities and cost as given in Table I are considered. The optimal machine assignment for a single part reconfigurable flow line allowing paralleling of similar machines for a single fixed demand period is illustrated. As shown in Fig. 3, the operation sequence of the product to be produced is assumed to be $3 \rightarrow 7 \rightarrow 15 \rightarrow 8 \rightarrow 1 \rightarrow 4 \rightarrow 17 \rightarrow 2 \rightarrow 9$ with a demand rate of 80 parts/hour. The number of stages is also assumed to be nine. The non-dominated solutions are obtained, which are seven in this case. The Pareto frontiers obtained through applying MOPSO are listed in the Table II. 
A number of trials were conducted to optimize the parameters involved in MOPSO. The final values of the parameters used in the experiment are, Swarm size $=200$, Number of iterations $=100$, Values of $C_{1}$ and $C_{2}$ are taken as 2 and the inertia weight $(w)$ is taken as 0.3 .

Table II: Non-dominated solutions obtained through MOPSO.

\begin{tabular}{|c|c|c|c|c|c|c|c|c|c|c|c|}
\hline \multirow{3}{*}{ \# } & \multirow{2}{*}{\multicolumn{9}{|c|}{$\begin{array}{l}\text { Solutions } \\
\text { Machine assigned/Number of machines }\end{array}$}} & \multicolumn{2}{|c|}{ Fitness values } \\
\hline & & & & & & & & & & \multirow{2}{*}{$\begin{array}{l}\text { Cost in } \\
10^{3} \text { of USD } \\
(F 1)\end{array}$} & \multirow{2}{*}{$\begin{array}{l}\text { Machine } \\
\text { Utilization } \\
(F 2)\end{array}$} \\
\hline & S-1 & $\mathrm{S}-2$ & S-3 & S-4 & S-5 & S-6 & $\mathrm{S}-7$ & $\mathrm{~S}-8$ & S-9 & & \\
\hline 1 & $13 / 4$ & $54 / 6$ & $22 / 6$ & $11 / 7$ & $51 / 5$ & $11 / 6$ & $23 / 4$ & $24 / 4$ & $12 / 4$ & 44640 & 0.9718 \\
\hline 2 & $13 / 4$ & $51 / 6$ & $32 / 4$ & $11 / 7$ & $51 / 5$ & $11 / 6$ & $23 / 4$ & $24 / 4$ & $12 / 4$ & 44830 & 0.9771 \\
\hline 3 & $13 / 4$ & $51 / 6$ & $22 / 6$ & $11 / 7$ & $51 / 5$ & $11 / 6$ & $23 / 4$ & $24 / 4$ & $53 / 3$ & 42860 & 0.9524 \\
\hline 4 & $13 / 4$ & $54 / 6$ & $32 / 4$ & $43 / 5$ & $51 / 5$ & $11 / 6$ & $23 / 4$ & $24 / 4$ & $12 / 4$ & 48230 & 0.9894 \\
\hline 5 & $13 / 4$ & $51 / 6$ & $22 / 6$ & $11 / 7$ & $51 / 5$ & $11 / 6$ & $42 / 3$ & $24 / 4$ & $53 / 3$ & 42800 & 0.9400 \\
\hline 6 & $13 / 4$ & $51 / 6$ & $22 / 6$ & $11 / 7$ & $51 / 5$ & $11 / 6$ & $23 / 4$ & $24 / 4$ & $12 / 4$ & 42990 & 0.9647 \\
\hline 7 & $13 / 4$ & $54 / 6$ & $32 / 4$ & $11 / 7$ & $51 / 5$ & $11 / 6$ & $23 / 4$ & $24 / 4$ & $12 / 4$ & 46480 & 0.9841 \\
\hline
\end{tabular}

\section{CONCLUSION AND FUTURE SCOPE}

In the present study a novel approach has been proposed to design the configuration of single part flow line in reconfigurable manufacturing system applying the multiple objective particle swarm optimization. In the present investigation a combinatorial problem of assigning the machine configuration in RMS has been attempted, which lies in the discrete and discontinuous search space. The search space has been reduced to the feasible region, improving the efficiency of the present approach. To extend the PSO for multiple objective optimization non-dominated sorting concept of NSGA-II has been adapted into the PSO algorithm. A fairly distributed Pareto front has been obtained, which can further be utilized for optimization of the reconfiguration cycle consisting of multiple time horizon. Considering the composite score of the solutions the decision manager may choose a suitable candidate among the Pareto frontiers to justify the objectives defined by the management along with the present market scenario. In future authors plan to study RMS for the multiple periods planning horizon.

\section{REFERENCES}

[1] Koren, Y.; Hiesel, U.; Jovane, F.; Moriwaki, T.; Pritschow, G.; Ulsoy, G.; Van-Brussel, H. (1999). Reconfigurable manufacturing systems, Annals of the CIRP, Vol. 48, No. 2, 527-540, doi:10.1016/S0007-8506(07)63232-6

[2] Mehrabi, M. G.; Ulsoy, A. G.; Koren, Y. (2000). Reconfigurable manufacturing systems: Key to future manufacturing, Journal of Intelligent Manufacturing, Vol. 11, No. 4, 403-419, doi:10.1023/A:1008930403506

[3] Urbani, A.; Molinari-Tosatti, L.; Pedrazzoli, P.; Fassi, I.; Boer, C. R. (2001). Flexibility and reconfigurability: An analytical approach and some examples, CIRP $1^{\text {st }}$ International Conference on Reconfigurable Manufacturing Systems, Ann Arbor, MI

[4] Pattanaik, L. N.; Jain, P. K.; Mehta, N. K. (2007). Cell formation in the presence of reconfigurable machines, International Journal of Advanced Manufacturing Technology, Vol. 34, No. 3-4, 335-345, doi:10.1007/s00170-006-0592-5

[5] Maier-Speredelozzi, V.; Koren, Y.; Hu, S. J. (2003). Convertibility measures for manufacturing systems, Annals of the CIRP, Vol. 52, No. 1, 367-370, doi:10.1016/S0007-8506(07)60603-9 
[6] Gumasta, K.; Gupta, S. K.; Benyoucef, L.; Tiwari, M. K. (2011). Developing a reconfigurability index using multi-attribute utility theory, International Journal of Production Research, Vol. 49, No. 6, 1669-1683, doi:10.1080/00207540903555536

[7] Dou, J. P.; Dai, X. Z.; Meng, Z. D. (2009). Graph theory-based approach to optimize singleproduct flow-line configurations of RMS, The International Journal of Advanced Manufacturing Technology, Vol. 41, No. 9, 916-931, doi:10.1007/s00170-008-1541-2

[8] Goyal, K. K.; Jain, P. K.; Jain, M. (2012). Optimal configuration selection for reconfigurable manufacturing system using NSGA II and TOPSIS, International Journal of Production Research, Vol. 50, No. 15, 4175-4191, doi:10.1080/00207543.2011.599345

[9] Saxena, L. K.; Jain, P. K. (2012). A model and optimisation approach for reconfigurable manufacturing system configuration design, International Journal of Production Research, Vol. 50, No. 12, 3359-3381, doi:10.1080/00207543.2011.578161

[10] Son, S. Y.; Olsen, T. L.; Derek, Y. (2001). An approach to scalability and line balancing for RMS, Integrated Manufacturing Systems, Vol. 12, No. 7, 500-511, doi:10.1108/ 09576060110407815

[11] Tang, L.; Yip-Hoi, D. M.; Wang, W.; Koren, Y. (2004). Concurrent line-balancing, equipment selection and throughput analysis for multi-part optimal line design, International Journal for Manufacturing Science and Production, Vol. 6, No. 1-2, 71-82

[12] Youssef, A. M. A.; ElMaraghy, H. A. (2006). Modelling and optimisation of multiple-aspect RMS configuration, International Journal of Production Research, Vol. 44, No. 22, 4929-4958, doi:10.1080/00207540600620955

[13] Youssef, A. M. A.; ElMaraghy, H. A. (2007). Optimal configuration selection for reconfigurable manufacturing systems, International Journal of Flexible Manufacturing Systems, Vol. 19, No. 2, 67-106, doi:10.1007/s10696-007-9020-x

[14] Kennedy, J.; Eberhart, R. (1995). Particle Swarm Optimization, Proceedings of the Fourth IEEE International Conference on Neural Networks, Perth, Australia, 1942-1948

[15] Eberhart, R. C.; Shi, Y. (2001). Particle swarm optimization: developments, applications and resources, Proceedings of IEEE Congress on Evolutionary Computation. Piscataway, NJ, Seoul, Korea, 81-86

[16] Shi, Y.; Eberhart, R. C. (1998). Parameter selection in particle swarm optimization, Evolutionary programming VII: Proceedings of EP 98, Springer, New York, 591-600

[17] Galzina, V.; Lujic, R.; Saric, T. (2012). Adaptive fuzzy particle swarm optimization for flowshop scheduling problem, Technical Gazette, Vol. 19, No. 1, 151-157

[18] Coello Coello, C. A.; Lechuga, M. S. (2002). MOPSO: A proposal for multiple objective particle swarm optimization, Proceedings of the Congress on Evolutionary Computation (CEC'2002), Honolulu, HI, Vol. 1, 1051-1056

[19] Coello Coello, C. A.; Pulido, G. T.; Lechuga, M. S. (2004). Handling multiple objectives with particle swarm optimization, IEEE Transactions on Evolutionary Computation, Vol. 8, No. 3, 256-279, doi:10.1109/TEVC.2004.826067

[20] Coello Coello, C. A.; Lamont, G. B.; Veldhuizen, D. A. V. (2007). Evolutionary Algorithms for Solving Multi-Objective Problems, $2^{\text {nd }}$ ed., Springer-Verlag, Berlin

[21] Salazar-Lechuga, M.; Rowe, J. E. (2005). Particle swarm optimization and fitness sharing to solve multi-objective optimization problems, Proceedings of IEEE Congress on Evolutionary Computation, Vol. 2, 1204-1211

[22] Li, X. A. (2003). Nondominated sorting particle swarm optimizer for multiobjective optimization, Proceedings of the Genetic and Evolutionary Computation Conference (GECCO 2003), Springer, Berlin, Part I, 37-48, doi:10.1007/3-540-45105-6 4

[23] Deb, K.; Samir, A.; Amrit, P.; Meyarivan, T. (2002). A fast elitist non-dominated sorting genetic algorithm for multi-objective optimization: NSGA-II, IEEE Transactions on Evolutionary Computation, Vol. 6, No. 2, 103-112, doi:10.1109/4235.996017

[24] Deb, K. (2001). Multi-objective optimization using evolutionary algorithm, John Wiley \& Sons Ltd, Chichester, UK 